\title{
Social interactions in male gerbils, Meriones unguiculatus: Ultrasounds and open-field behavior
}

\author{
J. M. GRAHAM and D. D. THIESSEN \\ University of Texas at Austin, Austin, Texas 78712
}

\begin{abstract}
Relationships among ultrasonic vocalizations and several other behaviors (aggression, locomotion, ventral-scent-gland marking, sniffing, and autogrooming) were examined during single 15-min interactions within 12 different pairs of adult male gerbils. Behaviors were assessed before and after social dominance was established within each pair. Ultrasound emissions were associated with fight severity $(\mathrm{r}=-.66)$, locomotion was associated with scent marking $(\mathrm{r}=.86)$ and with autogrooming $(\mathrm{r}=.68)$, and scent marking was associated with sniffing $(\mathrm{r}=.67)$. Dominant and subordinate animals showed different patterns of behavior both before and after dominance was established. Primary predictors of intrapair dominance were priority and latency of scent marking. After dominance was established, gerbils with the higher ranks showed significantly greater locomotion, sniffing, and autogrooming.
\end{abstract}

The Mongolian gerbil, like many other rodent species, vocalizes in the ultrasonic range during social interactions [see American Zoologist, 1979, 19(2), for a recent review]. One of the primary stimuli evoking ultrasounds is conspecific odors (Thiessen, Graham, \& Davenport, 1978). Males emit ultrasounds more frequently than do females, and socially dominant males are more responsive to biological odors than are subordinate males. High levels of ultrasonic emissions are induced with amphetamine, and lower levels follow social habituation (Thiessen et al., 1978, and unreported observations).

In the present experiment, associations were determined between ultrasonic emissions during malemale interactions and each of the following behaviors: intensity of aggression, locomotion, ventral scentgland marking, object sniffing, and autogrooming. Particular attention was given to detecting behaviors that predict levels of ultrasonic emission or change in these levels during formation of dominant-subordinate status.

\section{METHOD}

\section{Subjects}

Twenty-four male Mongolian gerbils, Meriones unguiculatus, approximately 200 days of age, served as subjects. Prior to the start of the experiment, the animals had been housed individually for at least 90 days. Purina Laboratory Chow and tap water were available ad lib. The animals were housed under a 12-h/12-h lightdark photoperiod.

\section{Apparatus}

The behavioral tests were conducted in a rectangular, clear, Plexiglas enclosure, $25 \times 38 \times 48 \mathrm{~cm}$. The floors and top of the

This study was supported by NIMH Grant 14076-13 awarded to D. D. Thiessen. test enclosure were removable for ease of cleaning. The floor had nine rectangular, plastic pegs, $2.5 \times 1.3 \times .64 \mathrm{~cm}$, which served as substrate for ventral scent marking, and the top had a $1.3-\mathrm{cm}$ hole in the center for the insertion of the ultrasound-detection microphone. The floor was divided symmetrically into a 3 by 3 grid, so that line crossings as a measure of locomotor activity could be determined. The behavior of each pair of animals in the experiment was videotaped using a video camera with zoom lens (Sony Model AVC 3260), video tape recorder (Sony Model AV 3650), and television monitor (Sony Model CKV 171). The test box was positioned in front of an oscilloscope (Tektronix Model 545), so that beam deflections corresponding to ultrasounds could be videotaped simultaneously with the behavior of the animals. The occurrence and duration of each behavioral event was recorded on an Esterline Angus recorder, with a chart speed of $61 \mathrm{~cm} / \mathrm{min}$. A darkroom timer was utilized to time the pair encounters. The Brüel and Kjaer ultrasonic recording equipment used in this experiment has been described in a previous report (Thiessen et al., 1978).

\section{Procedure}

The 24 animals were divided randomly into 12 pairs. Each animal was marked for identification by clipping a small amount of fur from the flank. Each pair was placed in the test box, and its behaviors, including ultrasounds, were videotaped for a single 15 min period. Between pair encounters, the test box was cleaned with a $75 \%$ solution of methanol and water

The occurrence and duration of the following behaviors for each member of a pair were recorded on separate channels of the event recorder: (1) locomotor activity (line crossing by the front two feet), (2) ventral scent marking (pressing the abdominal gland against a floor peg), (3) fighting (actual physical encounters), (4) autogrooming (full cleaning of the head), and (5) sniffing (nose orientation to an object accompanied by whisker movement).

The total number of ultrasounds for each $15-\mathrm{min}$ period was recorded; this total necessarily represents the number of sounds emitted by both animals. Overt fighting occurred in 11 of the 12 pair encounters. Aggression and dominance were assessed in these encounters independently of the other variables measured. The indices used in the assessments were drawn from measures of aggression and dominance developed by Scott (1966) and by Yahr, Coquelin, Martin, and Scouten (1977), and were as follows: (1) number of overt attacks; (2) number, distribution, and severity of injuries sustained; (3) attempts to escape the aggressor or the enclosure; and (4) defensive postures. Formation of dominance was 
clear in every pair in which fighting occurred. In addition, the amount of time (in seconds) that pairs spent fighting was used as the measure of severity. The frequency of or amount of time spent in other behaviors was summed across time for each pair, resulting in 12 scores for each measure.

\section{RESULTS}

The correlations among the various measures for the 12 pairs are shown in Table 1 . The only behavior that correlated significantly with the frequency of ultrasonic emissions was fight intensity. Locomotion correlated significantly both with the frequency of scent marking and with autogrooming, and sniffing correlated significantly with scent marking.

The significant inverse correlation $(-.66, \mathrm{df}=10$, $p<.05$ ) between ultrasounds and aggressive behavior is of particular interest, since ultrasound emission during paired encounters has been observed to be frequent during aggression for both gerbils and rats (Lore, Flannelly, \& Farina, 1976; Thiessen et al., 1978). A scatter plot of ultrasounds and fight severity is presented in Figure 1.

Correlations between the behavior of the dominant and subordinate animals were computed in order to determine the degree of dependency among their behaviors. Coefficients are reported in Table 2 . The behavior showing the greatest degree of association between the dominant and subordinate animals was locomotor activity, which is represented by a correlation coefficient of $.78(\mathrm{df}=10, \mathrm{p}<.003)$. This indicates that the activity level of one animal is a fairly good predictor of the activity level of its opponent and is independent of the absolute levels. Dominance was established on the average of $6.7( \pm 2.3)$ min into the test. Comparisons revealed that the animals that became dominant ventral-scent-marked more frequently than those that became subordinate before $[\mathrm{t}(10)=$ $2.52, \mathrm{p}<.05]$ and after $[\mathrm{t}(10)=2.80, \mathrm{p}<.05]$ dominance was established. The efficacy of using ventral scent marking as an indicant of dominance is shown by comparing the latency to the first scent mark for the dominant and subordinate animals. Animals which became dominant marked significantly earlier $[\mathrm{t}(10)=$ $2.44, \mathrm{p}<.05]$ than those which became subordinate. Dominant and subordinate animals did not show a

Table 1

Correlations of Behaviors Between Pairs of Competing Males

\begin{tabular}{lccccc}
\hline & & $\begin{array}{c}\text { Loco- } \\
\text { Sniffs }\end{array}$ & $\begin{array}{c}\text { Scent } \\
\text { motion }\end{array}$ & $\begin{array}{c}\text { Auto- } \\
\text { grooms }\end{array}$ & $\begin{array}{c}\text { Fight } \\
\text { Intensity }\end{array}$ \\
\hline Ultrasounds & -.49 & .30 & -.48 & -.42 & $-.66^{*}$ \\
Sniffs & & .50 & $.67^{*}$ & .07 & .09 \\
Locomotion & & & $.86^{\dagger}$ & $.68^{*}$ & -.11 \\
Scent Marks & & & & .35 & -.20 \\
Autogrooms & & & & & -.15 \\
\hline
\end{tabular}

${ }^{*} p<.02 . \quad t p<.001$

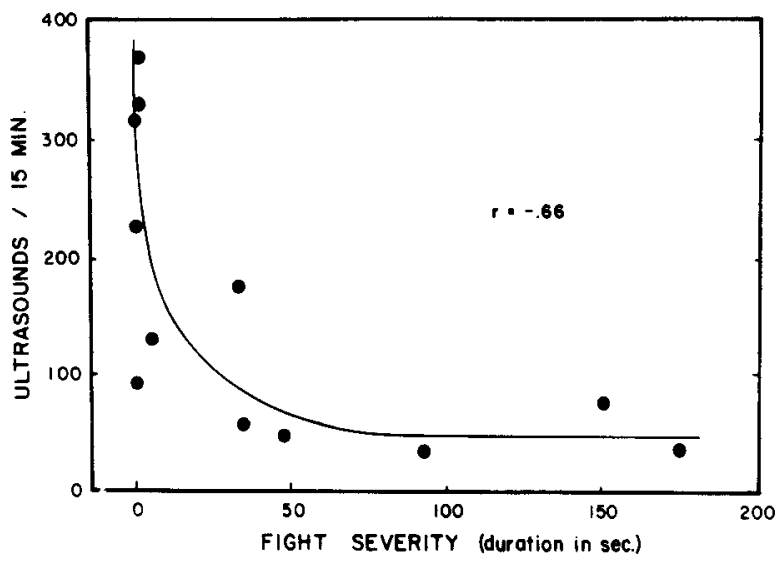

Figure 1. Relationship between ultrasound emissions in $15 \mathrm{~min}$ and fight severity.

Table 2

Correlations Between Dominant and Subordinate Males

\begin{tabular}{lc}
\hline \multicolumn{1}{c}{ Variable } & Correlation \\
\hline Locomoting & $.78^{*}$ \\
Sniffing & .29 \\
Scent Marking & .30 \\
Autogrooming & -.26 \\
\hline
\end{tabular}

${ }^{*} p<.003$.

differential amount of locomotor activity, sniffing, or grooming prior to the establishment of dominance; however, a differential pattern was observed after dominance was established. Animals which became dominant were significantly more active $[\mathrm{t}(10)=2.60$, $\mathrm{p}<.05]$, spent more time sniffing $[\mathrm{t}(10)=3.47$, $\mathrm{p}<.01]$, and self-groomed more frequently [t $(10)=$ $2.31, \mathrm{p}<.05]$ than those animals which became subordinate.

\section{DISCUSSION}

The 12 pairs of male gerbils engaged in a number of correlated behaviors during a 15-min encounter. Locomotion correlated significantly with the frequency of scent marking and autogrooming, and ventral scent marking correlated significantly with sniffing (Table 1). Apparently related facets of behavior characterize each pair. It is interesting that the only behavior that correlates with the frequency of ultrasonic emissions was time spent in aggressive contact (Figure 1). The finding suggests that ultrasounds may have a unique role in male-male aggression.

High levels of ultrasonic emission may inhibit fighting, as suggested for other rodent species (Lore et al., 1976; Nyby \& Whitney, 1978; Sales \& Pye, 1974). The correlation of -.66 between frequency of emission and fight severity in the gerbil seems to support 
this conclusion. There may be a more direct explanation, however, for these results. Possibly, high levels of ultrasonic emissions may simply be an index of a high level of behavioral arousal which is incompatible with fighting (Thiessen et al., 1978). Fights can begin only after locomotion has decreased substantially and gerbils begin to focus their attention on each other. Therefore, inactive animals, and those with low levels of ultrasound, may attend to each other sooner and begin to fight with a shorter latency. Indeed, the scatter plot of ultrasounds and aggression, which shows a quadratic function (Figure 1), suggests that male agonistic encounters may be typified either by high ultrasounds and low aggression or the reverse. In effect, the inverse relationship reported for ultrasound emission and fighting may be an indirect expression of arousal levels and locomotion, both of which are relevant to fighting. Species variations may, of course, account for the same relationship in other rodent species.

Fights generally start during a period of intense mutual sniffing and investigation, usually within the first $7 \mathrm{~min}$ of the encounter. Ultrasounds are generally absent at this time [e.g., the distribution of sniff frequency and ultrasonic emissions do not overlap; $\mathrm{t}(10)=2.78, \mathrm{p}<.01]$. The postfight period is characterized by a resumption of ultrasounds, which are mostly associated with movements of the dominant animal. After the fight, the dominant animal resumes ventral scent marking, often at a higher rate than before the fight, and autogrooms frequently. The subordinate animal scent-marks only rarely after the fight and shows a reduced rate of autogrooming. The pattern of locomotion and sniffing also changes. Before the fight, both animals characteristically display high levels of locomotion and object sniffing, but after the fight, the dominant animal is much more active and shows more sniffing.
Some measures can act as predictors of dominance status, while others correlate with the development of dominance status. Animals that were judged to be dominant scent-marked more frequently prior to fights and had the shorter latency to the first mark. Animals that became dominant thereafter showed increases in locomotion, sniffing, and autogrooming. Apparently some behaviors, such as scent marking, are predictors of social status, whereas other behaviors, such as locomotion, sniffing, and autogrooming are indicators of social status.

Individual locomotion within a pair is highly correlated (Table 2). Indeed, when the interaction is filmed and rerun at a fast speed, the interaction seems choreographed. Animals apparently attend to each other at all stages of interaction. The conclusion seems warranted that if any communication system is to be understood, all related behaviors must be studied within that same context.

\section{REFERENCES}

Lore, R., Flannelly, K., \& Farina, P. Ultrasounds produced by rats accompany decreases in intraspecific fighting. Aggressive Behavior, 1976, 2, 175-181.

NyBy, J., \& Whitney, G. Ultrasonic communication of adult myomorph rodents. Neuroscience Biobehavior Review, 1978, 2, $1-14$.

Sales, G., \& PYE, D. Ultrasonic communication by animals. London: Chapman and Hall, 1974.

ScotT, J. P. Agonistic behavior of mice and rats: A review. American Zoologist, 1966, 6, 683-701.

Thiessen, D. D., Graham, M., \& Davenport, R. Ultrasonic signaling in the gerbil (Meriones unguiculatus): Social interaction and of faction. Journal of Comparative and Physiological Psychology, 1978, 92, 1041-1049.

Yahr, P., Coquelin, A., Martin, A., \& Scouten, C. Effects of castration on aggression between male Mongolian gerbils. Behavioral Biology, 1977, 19, 189-205.

(Received for publication August 15, 1979; revision accepted April 23, 1980.) 